\title{
Does gastric acid suppression increase the risk of community-acquired pneumonia?
}

Laheij RJ, Sturkenboom MC, Hassing RJ, Dieleman J, Stricker BH, Jansen JB. Risk of community-acquired pneumonia and use of gastric acid-suppressive drugs. JAMA 2004;292(16):1955-60

Background: Dyspepsia is responsible for $7 \%$ of visits to family practitioners, ${ }^{1}$ and millions of Canadians have received 1 or both of the 2 main drug classes used to inhibit gastric acid secretion, histamine $\mathrm{H}_{2}$ receptor antagonists (H2RAs) and proton-pump inhibitors (PPIs). However, since an acidic gastric milieu serves as a defence against microorganisms, speculation exists that purposefully raising gastric $\mathrm{pH}$ levels may promote bacterial growth and thereby increase the risk of pneumonia.

Design: Researchers in the Netherlands examined the health care records of 364683 community-dwelling patients from 1995 to 2002 to explore the relation between acid suppression therapy and community-acquired pneumonia, both certain (a positive radiograph or culture) and probable (clinical symptoms only). Two observational designs were used. First, a retrospective cohort study estimated the relative risk of pneumonia among patients who received either an $\mathrm{H}_{2} \mathrm{RA}$ or PPI ( $n=19$ 459) compared with patients who did not receive such treatment ( $n=345224)$. Because these 2 groups were likely different in important but unmeasured ways, the investigators also performed a nested casecontrol analysis that focused on treated patients, in which cases with pneumonia $(n=475)$ were compared with cases without pneumonia $(n=4690)$ to examine the temporal relation to acid suppression therapy.

Results: Overall, 5.3\% of the study population received at least 1 prescription for an $\mathrm{H}_{2} \mathrm{RA}$ or PPI. Most received treatment empirically without endoscopy, but no information is presented to allow comparison with untreated patients. During 7562 person-years of treatment, 185 cases of pneumonia were identified, equivalent to a rate of about 1 case per 41 person-years of treatment or about 4.5 times greater than the rate among untreated patients. In the nested case-control analysis, pneumonia was weakly associated with active treatment (odds ratio $1.27 ; 95 \%$ confidence interval 1.06-1.54) but not with previous therapy. The risk during PPI therapy was not significantly greater than that during $\mathrm{H}_{2} \mathrm{RA}$ therapy, although the risk associated with higher PPI doses was almost double that with lower PPI doses.

Commentary: The study draws attention to an expensive and sometimes overprescribed class of drugs. ${ }^{2}$ The paper also illustrates the strengths of observational epidemiology while exposing its soft underbelly. If acid suppression does indeed cause community-acquired pneumonia, proving so with a clinical trial would be nearly impossible. We therefore rely on thoughtful studies such as this one to document an association (or lack thereof) between a drug and a suspected adverse event.

However, the study also reinforces the adage that association does not equal causation. The authors note that patients who receive $\mathrm{H} 2 \mathrm{RA}$ or PPI therapy are likely different from those who do not. They are more likely to smoke, consume alco- hol, be obese and have active gastroesophageal reflux. Each of these factors may bear more of a causal relation to pneumonia than acid suppression itself. The nested case-control analysis partially rebuts this argument, but the modest odds ratio of only 1.27 may simply reflect the possibility that patients with pneumonia are more likely than control subjects to require ongoing acid suppression therapy because of the coexistence of other unmeasured risk factors. This additional approach, therefore, does not fully resolve the potential confound between exposure and disease.

Practice implications: The results of this study suggest an association between the use of acid-suppressive drugs and community-acquired pneumonia. Whether these drugs actually cause pneumonia or simply represent a marker of risk for the disease is considerably less clear. On the basis of this study, it seems reasonable to consider withholding $\mathrm{H}_{2} \mathrm{RA}$ or PPI therapy in patients who experience recurrent pneumonia during treatment.

\section{Michael E. Detsky \\ Department of Medicine \\ David N. Juurlink \\ Department of Medicine \\ University of Toronto \\ Divisions of General Internal \\ Medicine, Clinical Pharmacology \\ and the Clinical Epidemiology Unit \\ Sunnybrook and Women's College \\ Health Sciences Centre \\ Toronto, Ont.}

\section{References}

1. Chiba N, Bernard L, O'Brien BJ, Goeree R, Hunt RH. A Canadian physician survey of dyspepsia management. Can 7 Gastroenterol 1998;12(1):83-90.

2. Hungin AP, Rubin GP, O'Flanagan $H$. Long-term prescribing of proton pump inhibitors in general practice. Br 7 Gen Pract 1999;49(443):451-3. 